\title{
Charge and Spin Transport in Magnetic Nanowires
}

\author{
N. Sedlmayr ${ }^{1}$, J. Berakdar², M. A. N. Araújo ${ }^{3}$, V. K. Dugaev ${ }^{4}$ and J. Barnaśs \\ ${ }^{1}$ Fachbereich Physik, Technische Universität Kaiserslautern \\ ${ }^{2}$ Department of Physics, Martin-Luther-Universität Halle-Wittenberg \\ ${ }^{3}$ Department of Physics, Évora University, Évora \\ CFIF, Insituto Superior Técnico, Lisbon \\ ${ }^{4}$ Department of Physics, Rzeszów University of Technology, Rzeszów \\ ${ }^{5}$ Department of Physics, Adam Mickiewicz University, Poznan \\ ${ }^{1,2}$ Germany \\ ${ }^{3}$ Portugal \\ 4,5 Poland
}

\section{Introduction}

The large interest in magnetic nanowires is mostly related to their possible application in magnetoelectronics. It was established experimentally that the magnetic domain walls (DWs) in very thin magnetic wires can affect substantially their resistance up to $1000 \%$ or even more (Chopra \& Hua (2002)), (Rüster et al. (2003)). On the other hand, the magnetic DWs in nanowires can be effectively controlled by weak external magnetic field and by electric current pulses. The latter effect of current-induced DW motion opens the way for various applications. One of the most impressive advances in this direction is the development of race-tracking memory devices for fast storage and reading of information (Parkin et al. (2008)). A review of the properties of magnetic nanowires with domain walls has been presented recently in Ref. (Kläui (2008)).

Several different phenomena have been in the scope of experimental and theoretical research, each of them being important for the use of magnetic nanowires in magnetoelectronics. The first one is the problem of transmission and reflection of electrons through the DW since this effect is mostly responsible for the magnetoresistivity. The other problem is related to the spin-transfer torque in magnetic nanowires and possible mechanisms of the current-induced DW motion. These mechanisms are responsible for effective current-induced control of the DW motion and correspondingly for current-induced variation of the resistance of magnetic nanowires. The third problem concerns the dynamics of the DWs motion since the dynamics are related to the possibility of fast current-induced control of the resistance.

In this Chapter we review our main results of theoretical investigations into charge and spin transport properties in magnetic nanowires, mostly using some simplified models. Solving the models one can understand better the underlying physical mechanisms of the spin and charge transport in magnetic nanowires, that can be also used in computer simulations, which should take into account real electronic and magnetic structure of a specific material. 
The details of our calculations and more information on the methods can be found in Refs.: (Dugaev et al. (2002)), (Dugaev et al. (2003)), (Dugaev et al. (2004)), (Dugaev et al. (2005)), (Araújo et al. (2006)), (Dugaev et al. (2006)), (Araújo et al. (2007)), (Dugaev et al. (2007)), (Sedlmayr et al. (2009)) and (Sedlmayr et al. (2010)).

\section{Reflection of electrons from the magnetic domain wall}

Spin-polarized electrons moving in a magnetic nanowire are scattered not only from phonons, impurities and defects like in the case of nonmagnetic materials, but also from any spatial variation of magnetization, which leads to scattering with the variation of electron momentum and electron spin. This problem was considered theoretically first in Refs. (Cabrera \& Falicov $(1974, a))$ and (Cabrera \& Falicov $(1974, b))$.

In the case of very slow variation of magnetization, when the adiabatic approximation is applied, the electron wavefunction can be described semiclassically. For the scattering from DW the condition of semiclassical approximation is $\lambda \ll L$, where $\lambda$ is the wavelength of electron and $L$ is the width of DW. This condition is usually fulfilled in relatively thick microwires. We consider first this case, which provides relatively weak electron backscattering, so that the effect of DW reduces to a small correction to the conductance.

\subsection{Transmission through a thick domain wall}

\subsubsection{Model}

Let us consider a ferromagnet with a nonuniform magnetization $\mathbf{M}(\mathbf{r})$. The one-particle Hamiltonian describing conduction electrons locally exchange-coupled to the magnetization $\mathbf{M}(\mathbf{r})$ takes the form

$$
H=-\frac{1}{2 m} \psi_{\alpha}^{\dagger} \Delta \psi_{\alpha}-J \psi_{\alpha}^{\dagger} \sigma_{\alpha \beta} \cdot \mathbf{M}(\mathbf{r}) \psi_{\beta}
$$

where $J$ is the exchange parameter, $\psi_{\alpha}$ and $\psi_{\alpha}^{\dagger}$ are the spinor field operators of electrons, $\sigma=$ $\left(\sigma_{x}, \sigma_{y}, \sigma_{z}\right)$ are the Pauli matrices, and we use the units with $\hbar=1$. Hamiltonian (1) can be used to describe electrons interacting with a domain wall in ferromagnetic metals or in magnetic semiconductors. The domain wall is modeled by a magnetization profile $\mathbf{M}(\mathbf{r})$, and we assume $|\mathbf{M}(\mathbf{r})|=$ const. Then we can write $J \mathbf{M}(\mathbf{r})=M \mathbf{n}(\mathbf{r})$, where $\mathbf{n}(\mathbf{r})$ is a unit vector field, and $M$ is measured in the energy units including the parameter $J$.

The first step is to perform a local unitary transformation [Tatara \& Fukuyama (1997)] $\psi \rightarrow$ $T(\mathbf{r}) \psi$, which removes the nonhomogeneity of $\mathbf{M}(\mathbf{r})$, that is $T(\mathbf{r})$ transforms the second term in Eq. (1) as $\psi^{\dagger} \sigma \cdot \mathbf{n}(\mathbf{r}) \psi \rightarrow \psi^{\dagger} \sigma_{z} \psi$. This transformation can be applied not only to a simple domain wall, but also to any other types of topological excitations in ferromagnetic systems, for instance helicoidal waves, skyrmions, and others. Applying this transformation to the kinetic part of Hamiltonian (1) one obtains

$$
\psi^{\dagger} \Delta \psi \rightarrow \psi^{\dagger}(\nabla+\mathbf{A}(\mathbf{r}))^{2} \psi,
$$

where the gauge field $\mathbf{A}(\mathbf{r})$ is given by $\mathbf{A}(\mathbf{r})=T^{\dagger}(\mathbf{r}) \nabla T(\mathbf{r})$. This gauge field $\mathbf{A}(\mathbf{r})$ is a matrix in the spin space.

Let us consider now a more specific case of a domain wall in a bulk system or a thick magnetic wire. We assume that the wall is translationally invariant in the $x-y$ plane: $\mathbf{M}(\mathbf{r}) \rightarrow \mathbf{M}(z)$ and $\mathbf{n}(\mathbf{r}) \rightarrow \mathbf{n}(z)$. For such a simple domain wall one can parameterize the unit vector field $\mathbf{n}(z)$ as

$$
\mathbf{n}(z)=(\sin \varphi(z), 0, \cos \varphi(z)),
$$


where the $z$-dependent phase $\varphi(z)$ determines the type of a domain wall. In this case the gauge field assumes the form

$$
\mathbf{A}(z)=\left(0,0,-\frac{i}{2} \sigma_{y} \varphi^{\prime}(z)\right)
$$

and the transformed Hamiltonian is

$$
H=-\frac{1}{2 m} \Delta+\frac{m \beta^{2}(z)}{2}-M \sigma_{z}+i \sigma_{y} \frac{\beta^{\prime}(z)}{2}+i \sigma_{y} \beta(z) \frac{\partial}{\partial z},
$$

where $\beta(z)=\varphi^{\prime}(z) / 2 m$ describes the local perturbation.

For a slowly varying smooth function $\varphi(z)$ (thick domain wall of width $L$ centered at $z=0$ ), the perturbation due to the domain wall is weak, and close to the center of the wall, $|z| \ll L$, the parameter $\beta(z)$ can be treated as a constant. If we assume the domain wall in the form of a kink, then $\varphi(z)=-(\pi / 2) \tanh (z / L)$, and the parameter $\beta(z)$ is given by

$$
\beta(z)=-\pi / 4 m L \cosh ^{2}(z / L) .
$$

\subsubsection{Semiclassical approximation}

In bulk magnetic metals like $\mathrm{Fe}, \mathrm{Ni}$ or $\mathrm{Co}$, the width $L$ of a magnetic domain wall is usually much larger then the electron Fermi wavelength $\lambda_{F}$. In such a case application of a semiclassical approximation can be justified (Cabrera \& Falicov $(1974, \mathrm{a})$; Cabrera \& Falicov $(1974, b))$. The dominant perturbation from the domain wall is then described by the term with $\beta(\partial / \partial z)$ in Eq. (5), since it is of order of $\beta k_{F}$. The term proportional to $\beta^{2}$ is smaller, while the term including $\beta^{\prime}(z)$ is of the order of $\beta / L$ and therefore can be neglected.

The Schrödinger's equation with Hamiltonian (5) has then the following semiclassical solutions $(i=1,2)$

$$
\psi_{i}(\boldsymbol{\rho}, z)=\frac{\exp ( \pm i \mathbf{q} \cdot \boldsymbol{\rho})}{\left[\varepsilon_{i}^{2}(z)+\beta^{2}(z) k_{i}^{2}(z)\right]^{1 / 2} k_{i}^{1 / 2}(z)}\left(\begin{array}{c}
\mp i \beta k_{i}(z) \\
\varepsilon_{i}(z)
\end{array}\right) \exp \left[ \pm i \int_{0}^{z} k_{i}(z) d z\right] .
$$

where $\rho=(x, y), \mathbf{q}$ is the momentum in the plane of the wall, the wavevector components normal to the wall (along the axis $z$ ) are given by

$$
k_{1,2}^{2}(z)=\kappa^{2}(z)+m^{2} \beta^{2}(z) \pm 2 m\left[M^{2}(z)+\beta^{2}(z) \kappa^{2}(z)\right]^{1 / 2},
$$

and

$$
\varepsilon_{i}(z)=\frac{k_{i}^{2}(z)}{2 m}+\frac{m \beta^{2}(z)}{2}-M(z)-\frac{\kappa^{2}(z)}{2 m},
$$

with $\kappa(z)$ defined as $\kappa^{2}(z)=2 m \varepsilon-q^{2}$. Obviously, there is no reflection from the wall in the semiclassical approximation.

\subsubsection{Scattering from the wall in Born approximation}

For the case of not too thin domain wall, the term proportional to $\beta(z) \partial / \partial z$ can be treated as a small perturbation and therefore the scattering from the wall can be evaluated within the Born approximation. The matrix elements of the $\left(k_{z} \uparrow\right) \rightarrow\left(k_{z}^{\prime} \downarrow\right)$ spin-flip scattering is given by

$$
V_{k_{z} k_{z}^{\prime}}=-\int_{-\infty}^{\infty} \mathrm{e}^{-i k_{z}^{\prime} z} \beta(z) \frac{d}{d z} \mathrm{e}^{i k_{z} z} d z
$$


Using (6) and calculating the integral we find

$$
V_{k_{z} k_{z}^{\prime}}=\frac{i \pi^{2} k_{z}\left(k_{z}^{\prime}-k_{z}\right) L}{4 m} \operatorname{csch}\left[\frac{\pi\left(k_{z}^{\prime}-k_{z}\right) L}{2}\right] \text {. }
$$

Correspondingly, the probability of backscattering $\left(k_{z}^{\prime}=-k_{z}\right)$ is

$$
W_{\text {back }} \equiv 2 \pi\left|V_{k_{z},-k_{z}}\right|^{2}=\frac{\pi^{5} k_{z}^{4} L^{2}}{2 m^{2}} \operatorname{csch}^{2}\left(\pi k_{z} L\right) .
$$

For $k_{z} L \gg 1$, from the last equation we find

$$
W_{\text {back }}=\frac{2 \pi^{5} k_{z}^{4} L^{2}}{m^{2}} \mathrm{e}^{-2 \pi k_{z} L} .
$$

Thus, the probability of the backscattering with simultaneous spin flip vanishes exponentially in the limit of $k_{F} L \gg 1$. The spin-conserving backscattering is determined by the term proportional to $\beta^{2}$ in the Hamiltonian (5). In the first approximation this term can be neglected as it gives a smaller contribution than the one proportional to $\beta(z) \partial / \partial z$.

The question arises, whether the Born approximation gives correct results for the problem under consideration. There are two general conditions for its applicability: $|U(z)| \ll 1 / m L^{2}$ or $|U(z)| \ll k / m L$, where $U(z)$ is the scattering potential. In the first case the Born approximation is good for arbitrary electron energy, whereas in the second one it is good only for fast electrons. Therefore, if we choose the limit $k_{F} L \gg 1$, then $|U(z)| \sim \beta k_{z} \sim\left(k_{z} / m L\right)$, and none of the conditions is satisfied. In the opposite case of a small domain-wall width, $k_{F} L \ll 1$, we have $|U(z)| \sim 1 / m L^{2}$ and the Born approximation is not justified again. Thus, the Born approximation can be used only for rough estimations. In the case under consideration, $k_{F} L \gg 1$, it just shows that the usual scattering from the wall is exponentially weak.

\subsection{Reflection from a thin domain wall}

\subsubsection{Model and scattering states}

Let us consider now a sharp domain wall assuming $\mathbf{M}(z)=M_{0} \mathbf{n}(z)=$ $\left[M_{0} \sin \varphi, 0, M_{0} \cos \varphi\right]$, where $\varphi(z)$ varies from zero to $\pi$ for $z$ changing from $z=-\infty$ to $z=+\infty$. When DW is laterally constrained, the number of quantum transport channels is limited. In the extreme case only a single conduction channel contributes. In such a situation, one can restrict considerations to a one-dimensional model, and write the Hamiltonian as

$$
H=-\frac{1}{2 m} \frac{d^{2}}{d z^{2}}-J M_{z}(z) \sigma_{z}-J M_{x}(z) \sigma_{x}
$$

Although this model describes only a one-channel quantum wire, it is sufficient to account qualitatively for some of the observations. Apart from this, it can be easily generalized to the case of a wire with more conduction channels.

In the following description we use the basis of scattering states. The asymptotic form of such states (taken sufficiently far from DW) can be written as

$$
\chi_{R \uparrow}(z)=\left\{\begin{array}{c}
\left(\begin{array}{c}
\mathrm{e}^{i k_{\uparrow} z}+r_{R \uparrow} \mathrm{e}^{-i k_{\uparrow} z} \\
r_{R \uparrow}^{f} \mathrm{e}^{-i k_{\downarrow} z}
\end{array}\right), z \ll-L, \\
\left(\begin{array}{c}
t_{R \uparrow} \mathrm{e}^{i k_{\downarrow} z} \\
t_{R \uparrow}^{f} \mathrm{e}^{i k_{\uparrow} z}
\end{array}\right), \\
z \gg L,
\end{array}\right.
$$


where $k_{\uparrow(\downarrow)}=\sqrt{2 m(E \pm M)}$, with $M=J M_{0}$, and $E$ denoting the electron energy. The scattering state (15) describes the electron wave in the spin majority channel incident from $z=-\infty$, which is partially reflected into the spin-majority and spin-minority channels, and also partially transmitted into these two channels. The coefficients $t_{R \uparrow}$ and $t_{R \uparrow}^{f}$ are the transmission amplitudes without and with spin reversal, respectively, whereas $r_{R \uparrow}$ and $r_{R \uparrow}^{f}$ are the relevant reflection amplitudes. It is worth to note that transmission from the spin-majority channel at $z<0$ to the spin-majority channel at $z>0$ requires spin reversal. The scattering states corresponding to the electron wave incident from $z=-\infty$ in the spin-minority channel have a similar form. Furthermore, scattering states describing electron waves incident from the right to the left take a similar form, as well.

In a general case (for $k_{F} L>1$ or $k_{F} L<1$ ) the transmission and reflection coefficients can be calculated numerically, as described below. When $k_{F} L \ll 1$, then the coefficients can be calculated analytically. Upon integrating the Schrödinger equation $H \psi=E \psi$ (with the Hamiltonian given by Eq. (14)) from $z=-\delta$ to $z=+\delta$, and assuming $L \ll \delta \ll k_{\uparrow(\downarrow)}^{-1}$, one obtains

$$
-\frac{1}{2 m}\left(\left.\frac{d \chi}{d z}\right|_{z=+\delta}-\left.\frac{d \chi}{d z}\right|_{z=-\delta}\right)-\lambda \sigma_{x} \chi(z=0)=0
$$

for each of the scattering states (for clarity of notation the index of the scattering states is omitted here), where

$$
\lambda \simeq J \int_{-\infty}^{\infty} d z M_{x}(z)
$$

Equation (16) has the form of a spin-dependent condition for electron transmission through a $\delta$-like potential barrier located at $z=0$. To obtain it we also used the condition $k_{\uparrow(\downarrow)} L \ll 1$, which is opposite to the condition used in the semiclassical approximation. The magnitude of the parameter $\lambda$ in Eq. (17) can be estimated as $\lambda \simeq J M_{0} L$.

Using the full set of scattering states together with the wave function continuity condition, one finds the transmission amplitudes

$$
\begin{aligned}
& t_{R \uparrow(\downarrow)}=t_{L \downarrow(\uparrow)}=\frac{2 v_{\uparrow(\downarrow)}\left(v_{\uparrow}+v_{\downarrow}\right)}{\left(v_{\uparrow}+v_{\downarrow}\right)^{2}+4 \lambda^{2}}, \\
& t_{R \uparrow(\downarrow)}^{f}=t_{L \downarrow(\uparrow)}^{f}=\frac{4 i \lambda v_{\uparrow(\downarrow)}}{\left(v_{\uparrow}+v_{\downarrow}\right)^{2}+4 \lambda^{2}},
\end{aligned}
$$

where $v_{\uparrow(\downarrow)}=k_{\uparrow(\downarrow)} / m$ denotes the electron velocity in the spin-majority (spin-minority) channel. According to (18), the magnitude of spin-flip transmission coefficients can be estimated as (for simplicity we omit here the eigenstate indices)

$$
\left|t^{f}\right|^{2} \sim\left(\frac{\lambda v}{v^{2}+\lambda^{2}}\right)^{2} \sim\left(\frac{M \varepsilon_{0}}{\varepsilon_{F} \varepsilon_{0}+M^{2}}\right)^{2}\left(k_{F} L\right)^{2},
$$

where $\varepsilon_{F}=k_{F}^{2} / 2 m$ and $\varepsilon_{0}=1 / m L^{2}$. For $k_{F} L \ll 1$ one finds $\varepsilon_{0} \gg \varepsilon_{F}$. Thus, taking $\varepsilon_{F} \sim M$, one obtains

$$
\left|t^{f}\right|^{2} \sim\left(\frac{M}{\varepsilon_{F}} k_{F} L\right)^{2} \ll 1
$$

Accordingly, a sharp domain wall can be considered as an effective barrier for the spin-flip transmission. On the other hand, the probability of spin conserving transmission is much 
larger, $\left|t / t^{f}\right|^{2} \sim \varepsilon_{F} \varepsilon_{0} / M^{2} \gg 1$. This means that electron spin does not follow adiabatically the magnetization direction when it propagates through the wall, but its orientation is rather fixed.

\subsubsection{Resistance of the domain wall}

To calculate the conductance of the system under consideration, one can start with the current operator $\hat{j}=e \psi^{\dagger}(z) \hat{v} \psi(z)$, where $\hat{v}$ is the velocity operator, whereas $\psi^{\dagger}(z)$ and $\psi(z)$ are the electron field operators taken in the spinor form. Using the expansion of $\psi(z)$ in the basis of scattering states (15) and carrying out quantum-mechanical averaging, one obtains the following formula for the current

$$
j=-i e \sum_{n} \int \frac{d k}{2 \pi} \int \frac{d \varepsilon}{2 \pi} \mathrm{e}^{i \varepsilon \eta} G_{n}(k, \varepsilon) \chi_{n}^{\dagger}(z) \hat{v} \chi_{n}(z),
$$

where $n$ is the index of scattering states $(n=R \uparrow, R \downarrow, L \uparrow$, and $L \downarrow)$ and $\eta=0^{+}$. The matrix elements of the velocity operator $\hat{v}=-(i / m) \partial / \partial z$ should be calculated in the basis of scattering states, and the retarded Green function $G_{n}(k, \varepsilon)$ in Eq. (21) is diagonal in this basis. Using Eq. (21) in the limit of small deviation from equilibrium, one obtains the Landauer formula for conductance, which takes into account the spin up and down channels

$$
G=\frac{e^{2}}{2 \pi \hbar}\left(\frac{v_{\downarrow}}{v_{\uparrow}}\left|t_{R \uparrow}\right|^{2}+\left|t_{R \uparrow}^{f}\right|^{2}+\frac{v_{\uparrow}}{v_{\downarrow}}\left|t_{R \downarrow}\right|^{2}+\left|t_{R \downarrow}^{f}\right|^{2}\right),
$$

where all the velocities and transmission coefficients are taken at the Fermi level.

For the thin domain wall, $k_{F \uparrow(\downarrow)} L \ll 1$, taking into account Eq. (15), one can write the conductance in the form

$$
G=\frac{4 e^{2}}{\pi \hbar} \frac{v_{\uparrow} v_{\downarrow}\left(v_{\uparrow}+v_{\downarrow}\right)^{2}+2 \lambda^{2}\left(v_{\uparrow}^{2}+v_{\downarrow}^{2}\right)}{\left[\left(v_{\uparrow}+v_{\downarrow}\right)^{2}+4 \lambda^{2}\right]^{2}} .
$$

In the limit of $v_{\uparrow}=v_{\downarrow}$ and $\lambda \rightarrow 0$, we obtain the conductance of a one-channel spin-degenerate wire, $G_{0}=e^{2} / \pi \hbar$. In the regime of ballistic transport $G_{0}$ is also the conductance of the system without the domain wall.

Variation of the conductance $G$ with the wall width $L$ (Fig. 1) was calculated from Eq. (22), with the transmission coefficients determined numerically. Thus, the results shown in Fig. 1, are valid for arbitrary value of $k_{F} L$. In the limit of $k_{F} L \ll 1$, the results shown in Fig. 1 coincide with those obtained from the formula (23).

The conductance of magnetic nanowire in the presence of a domain wall is substantially smaller than in the absence of the wall. Accordingly, the associated magnetoresistance can be large. For example, for $p=0.9$ in Fig. 1 the magnetoresistance is equal to about $70 \%$ (it corresponds to $G / G_{0}=0.6$ ). It should be noted that in magnetoresistance experiments on magnetic nanowires, for which the inequality $k_{F} L<1$ is fulfilled, one can have more than one domain walls. Accordingly, the magnetoresistance effect can be significantly enhanced.

In the case of strong polarization $\left(p>p_{c}\right)$ the main contribution to the conductance is associated with the spin-flip transmission through the domain wall, and the conductance increases monotonously with the width of the domain wall. 


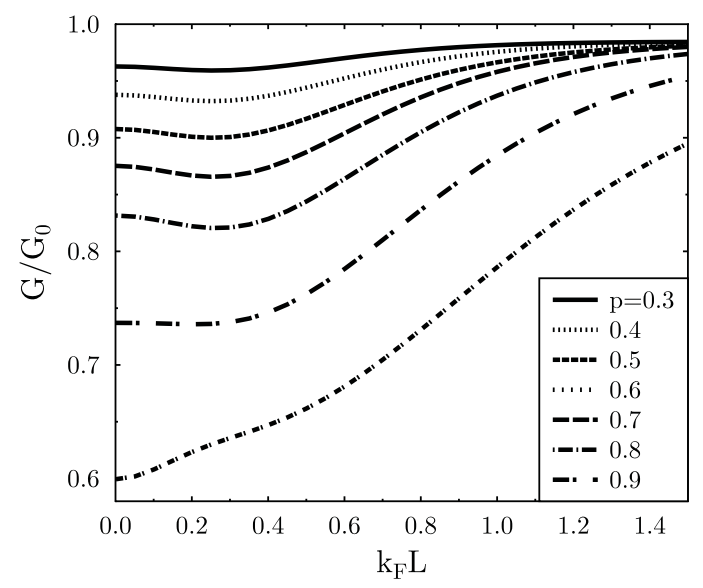

Fig. 1. Conductance of the domain wall as a function of DW width $L$ in a magnetic quantum wire for different values of the parameter $p=M / \varepsilon_{F}$.

\subsubsection{Spin current}

When the electric current is spin polarized and when there is some asymmetry between the two spin channels, the flow of charge is accompanied by a flow of spin (angular momentum). The z-component of the spin current can be calculated from the following definition of the corresponding spin-current operator (it is defined here as number of spins per unit time) $\hat{J}_{z}(z)=\frac{1}{2} \psi^{\dagger}(z)\left\{\sigma_{z}, \hat{v}\right\} \psi(z)$, which leads to the following average value

$$
J_{z}(z)=-i \sum_{n} \int \frac{d k}{2 \pi} \int \frac{d \varepsilon}{2 \pi} \mathrm{e}^{i \varepsilon \delta} G_{n}(k, \varepsilon) \chi_{n}^{\dagger}(z) \sigma_{z} \hat{v} \chi_{n}(z) .
$$

After carrying out the calculations similar to those described above for charge current, one arrives in the linear response regime (limit of small bias voltage $\mathcal{U}$ ) at the following formulas for the spin current $J_{z}$ :

$$
\begin{aligned}
& J_{z}(z<-L)=\frac{\mathcal{U}}{2 \pi \hbar}\left(\frac{v_{\downarrow}}{v_{\uparrow}}\left|t_{R \uparrow}\right|^{2}+\left|t_{R \uparrow}^{f}\right|^{2}-\frac{v_{\uparrow}}{v_{\downarrow}}\left|t_{R \downarrow}\right|^{2}-\left|t_{R \downarrow}^{f}\right|^{2}\right), \\
& J_{z}(z>L)=\frac{\mathcal{U}}{2 \pi \hbar}\left(\frac{v_{\downarrow}}{v_{\uparrow}}\left|t_{R \uparrow}\right|^{2}-\left|t_{R \uparrow}^{f}\right|^{2}-\frac{v_{\uparrow}}{v_{\downarrow}}\left|t_{R \downarrow}\right|^{2}+\left|t_{R \downarrow}^{f}\right|^{2}\right) .
\end{aligned}
$$

Using Eq. (15) we find

$$
J_{z}(z>L)=-\frac{8 e \mathcal{U}}{\pi} \frac{\lambda^{2}\left(v_{\uparrow}^{2}-v_{\downarrow}^{2}\right)}{\left[\left(v_{\uparrow}+v_{\downarrow}\right)^{2}+4 \lambda^{2}\right]^{2}}
$$

and $J_{z}(z<-L)=-J_{z}(z>L)$. The magnetic torque due to spin transfer to the magnetic system within the domain wall is determined by the non-conserved spin current

$$
T_{z}(U)=\frac{16 e \mathcal{U}}{\pi} \frac{\lambda^{2}\left(v_{\uparrow}^{2}-v_{\downarrow}^{2}\right)}{\left[\left(v_{\uparrow}+v_{\downarrow}\right)^{2}+4 \lambda^{2}\right]^{2}} .
$$


It should be noted that spin-flip scattering due to DW does not allow to separate spin channels like it was in the case for homogeneous ferromagnets. If we define now the spin conductance as $G_{z}^{s}=J_{z} / \mathcal{U}$, then one can write for $z>0$

$$
G_{z}^{s}=-\frac{8 e}{\pi} \frac{\lambda^{2}\left(v_{\uparrow}^{2}-v_{\downarrow}^{2}\right)}{\left[\left(v_{\uparrow}+v_{\downarrow}\right)^{2}+4 \lambda^{2}\right]^{2}} .
$$

Thus, $G_{z}^{s}$ is negative for $z>0$ and positive for $z<0$. In a nonmagnetic case we have $v_{\uparrow}=v_{\downarrow}$

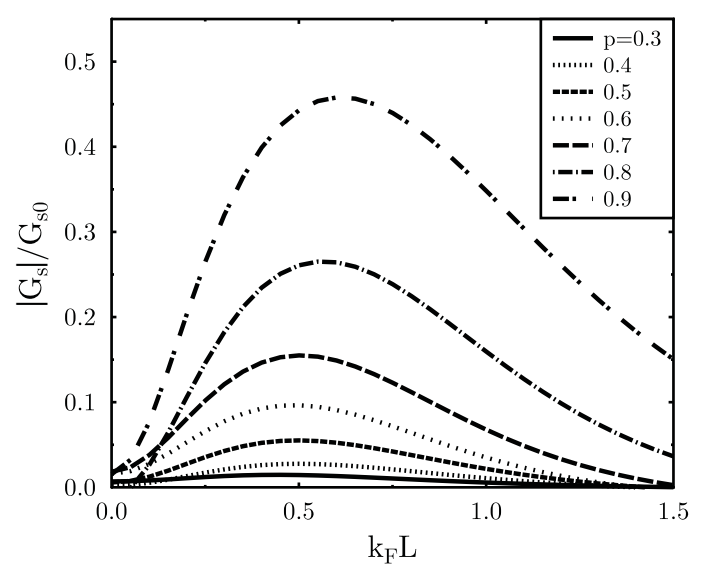

Fig. 2. Spin conductance of the wire with a domain wall as a function of DW width $L$ for different values of $p=M / \varepsilon_{F}$.

and therefore $G_{s}=0$. In the case considered here, $G_{s}=0$ when there is no DW in the wire. Let us introduce the spin conductance for one (spin-up) channel only, $G_{s 0}=e / 2 \pi$. The relative spin conductance in the presence of $D W, G_{S} / G_{s 0}$, calculated using Eq. (25) and with numerically found transmission coefficients, is shown in Fig. 2 as a function of the DW width $L$ and for indicated values of the parameter $p$. It corresponds to the spin current outside the region of the domain wall. The spin current inside the wall is not conserved due to the spin-flip transitions.

The nonzero spin current in a one-channel wire with domain wall is due to a difference in spin-flip transmissions for spin-up and spin-down channels: the corresponding transmission coefficient turns out to be larger for faster (majority) electrons.

\subsubsection{Spin polarization due to domain wall}

Spin dependent reflections from the wall lead to additional spin polarization of the system near the wall. The distribution of spin density created by the wall can be calculated using the basis of scattering states. The $z$-component of the spin density in the equilibrium situation $(\mathcal{U}=0)$ is

$$
S_{z}(z)=-i \sum_{n} \int \frac{d k}{2 \pi} \int \frac{d \varepsilon}{2 \pi} \mathrm{e}^{i \varepsilon \eta} G_{n}(k, \varepsilon) \chi_{n}^{\dagger}(z) \sigma_{z} \chi_{n}(z) .
$$


The above formula contains a constant part corresponding to the spin density in the absence of DW, as well as the $z$-dependent part $\delta S_{z}(z)$ created by the wall,

$$
\begin{aligned}
\delta S_{z}(z)= & \frac{1}{\pi} \int_{0}^{k_{F \uparrow}} d k r_{R \uparrow} \cos \left(2 k_{\uparrow} z\right)-\frac{1}{\pi} \int_{0}^{k_{F \downarrow}} d k r_{R \downarrow} \cos \left(2 k_{\downarrow} z\right), \quad(z<-L), \\
& =\frac{1}{\pi} \int_{0}^{k_{F \uparrow}} d k r_{L \uparrow} \cos \left(2 k_{\uparrow} z\right)-\frac{1}{\pi} \int_{0}^{k_{F \downarrow}} d k r_{L \downarrow} \cos \left(2 k_{\downarrow} z\right), \quad(z>L) .
\end{aligned}
$$

$>$ From (31) we infer that the spin density is an oscillating function of $z$. The spin dependent reflections from the wall create spatial oscillations of the electron spin density. These oscillations are similar to the Friedel oscillations of charge in a nonmagnetic metal. One should point out that in addition to the above calculated spin polarization, there is also a nonequilibrium spin polarization due to the current flowing through the system at $\mathcal{U} \neq 0$ (Ebels et al. (2000)).

\section{Spin quantum wells in magnetic nanowires}

Let us consider now the magnetic nanowire with a magnetization profile exhibiting two domain walls at a distance $2 d$. We assume that the magnetization $\mathbf{M}(z)$ in both DWs varies within the $x-z$ plane and the $z$ axis is along the wire. Thus, now $z$ is the easy axis, and the $x-z$ plane is the easy plane. We study the case where the thickness and the width of the nanowire are smaller than the carrier Fermi wavelength so that only one size quantized level (a single one-dimensional subband) is populated. The Hamiltonian describing independent carriers along the wire in the presence of magnetization field $\mathbf{M}(z)$ is given by Eq. (14).

We are interested in the case where the width $2 L$ of each DW is smaller than the carriers Fermi wavelength, $k_{F} L \ll 1$ and particularly when $k_{F} d \geq 1$ (otherwise the carriers are not influenced by the detailed topology of the DWs). For moderate carrier density the Fermi energy (chemical potential $\mu$ ) is in one of the magnetically split subbands. This case corresponds to a full spin polarization of the electron gas. The electron wave functions are

$$
\begin{aligned}
& \psi_{k}(z)=\left(e^{i k z}+r e^{-i k z}\right)|\uparrow\rangle+r_{f} e^{\kappa z}|\downarrow\rangle, \quad z<-d, \\
& \psi_{k}(z)=\left(A e^{\kappa z}+B e^{-\kappa z}\right)|\uparrow\rangle+\left(C e^{i k z}+D e^{-i k z}\right)|\downarrow\rangle, \quad|z|<d, \\
& \psi_{k}(z)=t e^{i k z}|\uparrow\rangle+t_{f} e^{-\kappa z}|\downarrow\rangle, \quad z>d,
\end{aligned}
$$

where $|\uparrow\rangle(|\downarrow\rangle)$ is the spin-up (spin-down) component of the carrier states, $k=[2 m(\varepsilon+$ $J M)]^{1 / 2}$, and $\kappa=[2 m(J M-\varepsilon)]^{1 / 2}$. The electron energy $\varepsilon$ is measured from the midpoint in between spin-up and spin-down band edges. The non-spin-flip (spin-flip) transmission and reflection coefficients $t$ and $r\left(t_{f}\right.$ and $\left.r_{f}\right)$ as well as the constants $A, B, C$ and $D$ have to be deduced from the solutions of Schrödinger's equation and from the continuity requirements. Equations (32)-(34) describe spin-up carriers incoming from the left, being transmitted and reflected from the double DW structure into waves with the same or opposite spin polarizations with a subsequent decay of the spin-down part of the wave function.

To determine the unknown coefficients in Eqs. (32)-(34) we utilize the wave function continuity at $z= \pm d$, i.e.

$$
\frac{1}{2 m}\left(\left.\frac{d \psi_{k}}{d z}\right|_{z=-d+L}-\left.\frac{d \psi_{k}}{d z}\right|_{z=-d-L}\right)+\lambda \sigma_{x} \psi_{k}(-d)=0,
$$


where

$$
\lambda \simeq J \int_{-d-L}^{-d+L} d z M_{x}(z) \simeq 2 J M L
$$

Similar equation holds for $z=d$. The boundary conditions at $z= \pm d$ (eight equations for the spinor components) define all the coefficients.

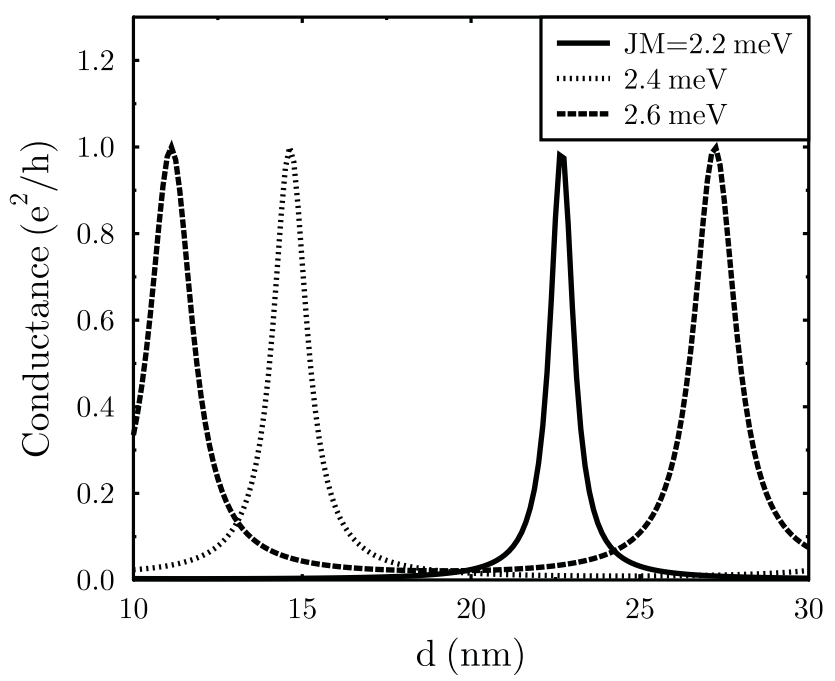

Fig. 3. Conductance as a function of the spin quantum well width for different values of the magnetization $M$.

For a physical insight into the results one can inspect the limiting case of $\Delta=4 m J M L=0$, where no spin-flip transitions occur at the DW. Correspondingly, only spin-up electrons tunnel through the barrier. Then the standard formula for barrier tunnelling

$$
t=2 i k \kappa e^{-2 i k d}\left[2 i k \kappa \cosh (2 \kappa d)+\left(k^{2}-\kappa^{2}\right) \sinh (2 \kappa d)\right]^{-1}
$$

can be easily retrieved. The corresponding contribution to the conductance is very small, and for $\kappa d \gg 1$ it can be completely neglected. The spin-down electrons are localized within the spin quantum well. One can find a symmetric solution with $r_{f}=t_{f}$ corresponding to a localized state with the wave vector $k$ and obeying the relation $\tan k d=\kappa / k$. The antisymmetric solution for such $k$ (with $r_{f}=-t_{f}$ ) satisfies the equation $\tan k d=-\kappa / k$. When the distance $d$ between the DWs is varied the energetic positions of the size-quantized levels within the well are shifted. For certain values of $d$ the energy of the localized states within the well coincides with the Fermi level. Thus, if the spin-mixing amplitude is finite (i.e. $\Delta \neq 0$ ) we expect spin-up carriers to transverse resonantly the DWs. It is important to note that when $\Delta \neq 0$, the aforementioned localized spin-down states turn quasi stationary with a finite decay width $\Gamma=1 / \tau \sim\left|t_{f}\right|^{2}$, where $\tau$ is the life time of these quasilocalized states. The parameter $\left|t_{f}\right|$ is controllable, e.g. by changing the parameters of the DWs.

In the regime of a linear response the conductance of the nanowire is determined by 


$$
G=\frac{e^{2}}{2 \pi}|t(\varepsilon=\mu)|^{2} .
$$

Using this relation one calculates the variation of the conductance $G$ with the DWs distance $2 d$ for several values of the magnetization $M$. The conductance shown in Fig. 3 exhibits narrow resonance peaks corresponding to those values of $d$ at which a quasi-discrete, size-quantized level coincides with the Fermi energy. The effective barrier created by the DWs is basically transparent at the conductance peak. The width of the resonance peaks is related to the life time $\tau$ of the quasi-stationary spin-well states, and is determined by the spin-mixing mechanism mentioned above (and specifically by $\left|t_{f}\right|$ ). The strength of spin mixing, and hence the width of the resonance peaks, can be controlled, e.g., by varying the width $L$ of the DWs. Decreasing the spin-mixing parameter $\Delta=4 m J M L$, the life time of the localized spin quantum-well states increases and the conductance resonance peaks become correspondingly narrower. The energetic positions of the quasi discrete levels depend also on the parameters $\Delta$ and $d$. This results in a slight shift of the resonance positions when changing $L$. Experimentally the Fermi level position can be shifted by electrically gating the whole structure. In this case, the resonance conductance peaks occur as a function of the gate voltage for a fixed distance between the DWs.

The presented calculations are valid for $d \ll L_{e}$, where $L_{e}$ is the decoherence length. Taking into account the decoherence effects, one expects that the double DWs resistance in this limit to be the sum of resistances of the individual DWs.

By gating the structure one can tune the Fermi energy and manipulate the spin density. For an experimental verification we note that spin-density modulations can be imaged with a sub nanometer resolution using spin-polarized scanning tunneling microscopy. The extreme sensitivity of the conductance to the inter-walls distance can be used to identify the relative position of the DWs.

\section{Influence of electron-electron interactions}

\subsection{Thin domain walls}

Now we consider quasi-one-dimensional magnetic nanowire with the domain wall taking into account the electron-electron interaction. This interaction is important at very low temperatures, and it can affect dramatically the charge and spin transport. We assume that the wire lies along $z$ axis, and the domain wall is centered at $z=0$. Its space modulation is described by $\mathbf{M}(\mathbf{r})$. Assuming $\mathbf{M}(\mathbf{r})$ to lie in the $x z$ plane, and the domain wall to be thinner than the Fermi wavelength, we write the single-particle Hamiltonian as

$$
H_{0}=-\frac{1}{2 m} \frac{d^{2}}{d z^{2}}+V \delta(z)+J M_{z}(z) \sigma_{z}+\lambda \delta(z) \sigma_{x},
$$

where $V$ is a potential scattering term that has been added for the sake of generality. The electron wavevector in each domain is related to the energy $E$ by $k=\left[2 m\left(E \pm J M_{0}\right)\right]^{1 / 2}$. The electron gas in the negative semi-axis $(z<0)$ is predominantly $\uparrow$-spin. An electron incident from the left with the momentum $k$ and spin $\uparrow$ (or $\downarrow$ ) can be transmitted to the positive semi-axis while preserving its spin, but the energy conservation requires the momentum to change from $k$ to $k_{-}$(or $k_{+}$), the latter being defined as a function of $k$ as $k_{ \pm}=\left(k^{2} \pm 4 m J M_{0}\right)^{1 / 2}$. If the transmission occurs with spin reversal, the momentum $k$ is not changed. 
Using the scattering states relevant to the problem, in the form of equation (15), one can easily find the scattering amplitudes for electrons incident from $z=-\infty$ :

$$
\begin{aligned}
& t_{\uparrow, \downarrow}(k)=\frac{2\left(v+v_{\mp}+2 i V\right) v}{\left(v+v_{\mp}+2 i V\right)^{2}+4 \lambda^{2}}=r_{\uparrow, \downarrow}(k)+1, \\
& t_{\uparrow, \downarrow}^{f}(k)=\frac{4 i \lambda v}{\left(v+v_{\mp}+2 i V\right)^{2}+4 \lambda^{2}}=r_{\uparrow, \downarrow}^{f}(k),
\end{aligned}
$$

where the velocities $v_{ \pm}=k_{ \pm} / m$. This is simply a generalization of equation (18) including now the potential scattering. These scattering amplitudes can be viewed as zero order with respect to the electron-electron interaction. Analogously one can determine the scattering states and amplitudes corresponding to the waves incident from $+\infty$.

The electron interactions are modeled by adding the following term to the Hamiltonian:

$$
\hat{H}_{\text {int }}=g_{1, \alpha, \beta} \int \frac{d k_{1} d q}{(2 \pi)^{2}} a_{k_{1}, \alpha}^{+} b_{k_{2}, \beta}^{+} a_{k_{2}+q, \beta} b_{k_{1}-q, \alpha}+g_{2, \alpha, \beta} \int \frac{d k_{1} d q}{(2 \pi)^{2}} a_{k_{1}, \alpha}^{+} b_{k_{2}, \beta}^{+} b_{k_{2}+q, \beta} a_{k_{1}-q, \alpha},
$$

where the coupling constants $g_{1}$ and $g_{2}$ describe back and forward scattering processes between opposite moving electrons, respectively. We make use of field operators describing right $\left(\hat{a}_{q \sigma}\right)$ and left $\left(\hat{b}_{q \sigma}\right)$ moving plane-wave states. The Greek indices denote spin, and the summation over repeated indices is implied. Since the Fermi momentum depends on spin, we allow for the dependence of $g$ on the spins of the interacting particles. We therefore distinguish between $g_{1 \uparrow}, g_{1 \downarrow} g_{1 \perp}$ and $g_{2 \uparrow}, g_{2 \downarrow}, g_{2 \perp}$. The forward scattering process between particles which move in the same direction will not affect the transmission amplitudes, although it will renormalize the Fermi velocity (Matveev et al. (1993)). This effect is equivalent to an effective mass renormalization, and the electrons with different spin orientations may turn out to have different effective masses.

The Hamiltonian terms (42) produce corrections, in first order of perturbation theory, to the scattering amplitudes, which we denote, for example, as $\delta t_{\uparrow}^{(f)}, \delta r_{\uparrow}^{(f)}$. If the bandwidth is $D$, and the energy of the scattered electron, $\epsilon$, approaches the Fermi level $(\epsilon \rightarrow 0)$ then these corrections diverge logarithmically as $\log \left(|\epsilon| / D_{0}\right)$ (Matveev et al. (1993)) and are dealt with in a poor man's renormalization method, which works as follows. The bandwidth $D$ is reduced step by step and such removal of states near the band edge is compensated by a renormalization of $t_{\uparrow}$. The problem of working out the first order perturbative corrections to the amplitudes is repeated for each new bandwidth with renormalized scattering amplitudes. Noting that $t_{\uparrow}+\delta t_{\uparrow}$ remains invariant as $D$ is reduced, one finds the following renormalization group differential equation:

$$
d t_{\uparrow}+\frac{\partial \delta t_{\uparrow}}{\partial D} d D=0
$$

and analogous equations for all the other scattering amplitudes. The bandwidth is progressively reduced from $D_{0}$ to $\left|\epsilon^{\prime}\right|$ (which will eventually be taken as temperature $\left|\epsilon^{\prime}\right|=T$ ). Then the scaling equations for the transmission amplitudes can be found Araújo et al. (2006) and solved in the vicinity of some fixed points. The nature and location of fixed point are determined by some combination of initial coupling constants in (42). In our analysis they are the interaction parameters $g_{\uparrow}, g_{\downarrow}$ and $g_{\perp}$, which are defined as follows: 


$$
\begin{aligned}
& \frac{g_{2 \uparrow}-g_{1 \uparrow}}{4 h v_{F+}}=g_{\uparrow}, \\
& \frac{g_{2 \downarrow}-g_{1 \downarrow}}{4 h v_{F-}}=g_{\downarrow}, \\
& \frac{g_{2 \perp}}{2 h\left(v_{F+}+v_{F-}\right)}=g_{\perp},
\end{aligned}
$$

where $v_{F-}, v_{F+}$ are the Fermi velocities of spin minority and spin majority electrons, respectively.

For repulsive e-e interactions the system flows to fixed points where it is an insulator. The fixed point where $\left|r_{\sigma}\right| \rightarrow 0$ is attained when $2 g_{\perp}-g_{\uparrow}-g_{\downarrow}>0$, in which case $\left|r_{\uparrow}^{f}\right| \rightarrow$ $\sqrt{v_{F+} / v_{F-}}$. The reflection coefficient with spin reversal depends on temperature as:

$$
\mathcal{R}_{\uparrow}^{f}(T)=\frac{v_{F-}}{v_{F+}}\left|r_{\uparrow}^{f}\right|^{2}=\frac{\frac{\mathcal{R}_{\uparrow, 0}^{f}}{1-\mathcal{R}_{\uparrow, 0}^{f}}\left(\frac{T}{D_{0}}\right)^{2\left(g_{\uparrow}+g_{\downarrow}-2 g_{\perp}\right)}}{1+\frac{\mathcal{R}_{\uparrow, 0}^{f}}{1-\mathcal{R}_{\uparrow, 0}^{f}}\left(\frac{T}{D_{0}}\right)^{2\left(g_{\uparrow}+g_{\downarrow}-2 g_{\perp}\right)}},
$$

where $\mathcal{R}_{\uparrow, 0}^{f}$ and is a constant equal to the reflection coefficient for non-interacting electrons. It is easily seen that when $2 g_{\perp}-g_{\uparrow}-g_{\downarrow}>0$, then $\mathcal{R}_{\uparrow}^{f}(T) \rightarrow 1$ as $T \rightarrow 0$. The domain wall becomes insulating. It reflects all incident electrons while reversing their spin. Therefore, such a DW may be considered as a perfect spin-flip reflector at zero temperature. In order to find the low $T$ behavior of transmissions we put $r_{\uparrow}=r_{\downarrow}=0$ in the renormalization group equations and obtain $\left|t_{\uparrow}\right| \sim\left|t_{\uparrow}^{f}\right| \sim\left|t_{\downarrow}^{f}\right| \sim T^{2 g_{\perp}}$.

In the regime where $g_{\uparrow}+g_{\downarrow}-2 g_{\perp}>0$ we have $\mathcal{R}_{\uparrow}^{f}(T) \rightarrow 0, \mathcal{R}_{\uparrow}(T) \rightarrow 1$. So, the domain wall reflects all incident electrons while preserving their spin. For the transmission amplitudes we can obtain: $\left|t_{\uparrow}\right| \sim T^{g_{\uparrow}+g_{\downarrow}},\left|t_{\uparrow}^{f}\right| \sim T^{2 g_{\uparrow}}$, and $\left|t_{\downarrow}^{f}\right| \sim T^{2 g_{\downarrow}}$.

If $g_{\uparrow}+g_{\downarrow}-2 g_{\perp}=0$ then both $\mathcal{R}_{\uparrow}^{f}(T)$ and $\mathcal{R}_{\uparrow}(\mathcal{T})$ tend to finite values.

Zero temperature fixed points corresponding to the transparent domain wall can be achieved when the interaction constants are all negative, and that corresponds to an attractive electron-electron interaction. The reflection coefficients vanish under scaling as powers of temperature. If some of the interaction constants are positive and the others negative, the situation becomes more complex. We now describe several possible situations for the case $V=0$ in the Hamiltonian (39).

If $g_{\uparrow}, g_{\downarrow}>0, g_{\perp}<0$ then the system flows to the fixed point $r_{\uparrow}=r_{\downarrow}=-1$ with all other amplitudes vanishing. The low- $T$ behavior of the transmission can be found as $\left|t_{\uparrow}\right| \sim T^{g \uparrow}+g_{\downarrow}$, $\left|t_{\uparrow}^{f}\right| \sim T^{2 g_{\uparrow}}$, and $\left|t_{\downarrow}^{f}\right| \sim T^{2 g_{\downarrow}}$. For $r_{\uparrow}^{f}$, one finds $r_{\uparrow}^{f} \sim T^{g_{\uparrow}+g_{\downarrow}-2 g_{\perp}}$, therefore we must have $g_{\uparrow}+g_{\downarrow}-2 g_{\perp}>0$ in order for $r_{\uparrow}^{f} \rightarrow 0$.

In the case of $g_{\uparrow}, g_{\downarrow}<0, g_{\perp}>0$ the system flows to the perfect spin-flip reflector fixed point $\left|r_{\uparrow}^{f}\right|=\sqrt{v_{F+} / v_{F-}}$ with all other amplitudes vanishing.

In the case of $g_{\uparrow}>0, g_{\downarrow}<0$ and for negative or small positive $g_{\perp}$, the system flows to a fixed point where $\left|t_{\downarrow}^{f}\right|=1, r_{\uparrow}=-1$. The wall transmits all spin-down particles with a spin-flip and 
reflects all spin-up particles. The exponents for the transmission amplitudes are $\left|t_{\uparrow}\right| \sim T^{g_{\uparrow}}$ and $\left|t_{\uparrow}^{f}\right| \sim T^{2 g_{\uparrow}}$.

When $g_{\uparrow}<0$ and $g_{\downarrow}>0$, the situation is analogous to the previous one. For negative or small positive $g_{\perp}$ the system flows to a fixed point where $\left|t_{\uparrow}^{f}\right|=1, r_{\downarrow}=-1$ with all the other amplitudes vanishing. The wall transmits all spin-up particles with a spin-flip and reflects all spin-down particles. The exponents for transmission amplitudes are $\left|t_{\uparrow}\right| \sim T^{g \downarrow},\left|t_{\downarrow}^{f}\right| \sim T^{2 g_{\downarrow}}$. Thus, we identified possible fixed points corresponding to (i) perfectly insulating wall (with or without complete spin reversal), and (ii) transparent wall. In any case, the electron electron interaction changes completely the transport through the domain wall at sufficiently low temperatures and may in some cases suppress charge transport without suppressing spin current. These results may account for a huge magnetoresistance associated with a domain wall in ballistic nanocontacs.

\subsection{Thick domain walls}

Electron-electron correlations play also role in the case of thicker domain walls (Sedlmayr et al. (2011)). Starting from equation (1), with an additional short range interaction $H_{I}$ similar to equation (43), one can again make the gauge transformation, equations (2)-(5). This gauge transformation allows one to linearize the problem around the spin-split Fermi points and hence use the standard technique of bosonization. After this gauge transformation the interaction is left unaffected, the magnetization is locally rotated to a Zeeman term, and the kinetic energy operator introduces a new potential when it acts on the local rotation:

$$
\begin{aligned}
& H=\sum_{\sigma} \int \mathrm{d} z \psi_{\sigma}^{\dagger}(z)\left[-\frac{1}{2 m} \partial_{z}^{2}-J M_{s} \sigma_{\sigma \sigma}^{z}-\mu\right] \psi_{\sigma}(z)+H_{I}+H_{w} \\
& H_{w}=-\frac{1}{2 m} \sum_{\sigma \sigma^{\prime}} \int \mathrm{d} z \psi_{\sigma}^{\dagger}(z)\left[i \varphi^{\prime}(z) \sigma_{\sigma \sigma^{\prime}}^{x} \partial_{z}+\frac{i \varphi^{\prime \prime}(z)}{2} \sigma_{\sigma \sigma^{\prime}}^{x}-\frac{1}{4}\left[\varphi^{\prime}(z)\right] 2 \delta_{\sigma \sigma^{\prime}}\right] \psi_{\sigma^{\prime}}(z) .
\end{aligned}
$$

$\psi_{\sigma}^{\dagger}(z)$ is a creation operator for an electron of spin $\sigma$ at a position $z$ in the wire. The gauge potential $H_{w}$ can be approximated if we assume that the Fermi wavelength is much smaller than the domain wall width $\lambda_{F} \ll L$. In this case only the first term of equation (48) is relevant, the next two terms are of order $\left(\lambda_{F} / L\right)^{2}$. The next step is linearization via the ansatz $\psi_{\sigma}(z)=$ $e^{i k_{F \sigma} x} \psi_{\sigma+}(z)+e^{-i k_{F \sigma} x} \psi_{\sigma-}(z)$, where $k_{F \sigma}=\sqrt{2 m\left(\varepsilon_{F} \pm J M\right)}$. The + and - indices denote the chiral right and left moving electrons respectively. The interaction $H_{I}$ can be decomposed into spin parallel and spin perpendicular components which, when suppressing the spatial indices and defining the local density $\rho_{\sigma \pm}=\psi_{\sigma \pm}^{\dagger} \psi_{\sigma \pm}$, can be written as

$$
\begin{aligned}
& H_{2}=\sum_{\sigma, r= \pm} \int \mathrm{d} z\left[\frac{g_{2} \| \sigma}{2} \rho_{\sigma r} \rho_{\sigma-r}+\frac{g_{2 \perp}}{2} \rho_{\sigma r} \rho_{\bar{\sigma}-r}\right] \\
& H_{4}=\sum_{\sigma, r= \pm} \int \mathrm{d} z\left[\frac{g_{4 \| \sigma}}{2} \rho_{\sigma r} \rho_{\sigma r}+\frac{g_{4 \perp}}{2} \rho_{\sigma r} \rho_{\bar{\sigma} r}\right] \\
& H_{1}=\sum_{\sigma, r= \pm} \int \mathrm{d} z\left[-\frac{g_{1 \| \sigma}}{2} \rho_{\sigma r} \rho_{\sigma-r}+\frac{g_{1 \perp \sigma}}{2} e^{2 i z\left(k_{\left.\bar{F}-k_{F \sigma}\right)}\right.} \psi_{\sigma r}^{\dagger} \psi_{\bar{\sigma}-r}^{\dagger} \psi_{\bar{\sigma} r} \psi_{\sigma-r}\right] .
\end{aligned}
$$

Umklapp processes scattering two left movers into right movers and vice versa are always neglected here due to the non-commensurate nature of the Fermi wavevectors. 
This fermionic model can now be bosonized (Penc et al. (1993)). Two adjoint bosonic fields are introduced which can be defined by the vertex operator

$$
\psi_{\sigma r}(z)=\frac{1}{\sqrt{2 \pi \alpha}} e^{-i \sqrt{\pi}\left[\theta_{\sigma}(z)-r \phi_{\sigma}(z)\right]}
$$

These bosonic fields satisfy $\left[\phi_{\sigma}(z), \Pi_{\sigma}\left(z^{\prime}\right)\right]=i \delta\left(z-z^{\prime}\right)$ where $\Pi_{\sigma}(z)=\partial_{y} \theta_{\sigma}(z)$. Despite the absence of spin-charge separation, due to the spin-split bands, we will use the spin and charge representation as it conveniently represents the scattering terms. Defining $\phi_{c, s}(z)=$ $\left[\phi_{1}(z) \pm \phi_{2}(z)\right] / \sqrt{2}$ (and similar for the $\theta_{c, s}(z)$ fields) gives us for the quadratic part of the bosonic Hamiltonian

$$
\begin{aligned}
H_{q}=\int \mathrm{d} z & {\left[\left[\partial_{z} \theta_{c}(z)\right] 2 \frac{v_{c} K_{c}}{2}+\left[\partial_{z} \theta_{s}(z)\right] 2 \frac{v_{s} K_{s}}{2}+\left[\partial_{z} \theta_{c}(z) \partial_{z} \theta_{s}(z)\right] v_{a}\right.} \\
+ & {\left.\left[\partial_{z} \phi_{c}(z)\right] 2 \frac{v_{c}}{2 K_{c}}+\left[\partial_{z} \phi_{s}(z)\right] 2 \frac{v_{s}}{2 K_{s}}+\left[\partial_{z} \phi_{c}(z) \partial_{z} \phi_{s}(z)\right] v_{b}\right] . }
\end{aligned}
$$

$K_{s}$ and $K_{c}$ are the spin and charge Luttinger parameters. $v_{a}$ and $v_{b}$ describe the coupling between the spin and charge sectors. These parameters are functions of the interaction strengths and Fermi velocities. This quadratic Hamiltonian contains all of the quartic fermionic interaction terms except for $g_{1 \perp \sigma}$.

The final bosonic Hamiltonian is $H=H_{q}+H_{1 \perp}+H_{w}^{f}+H_{w}^{b}$ where

$$
\begin{aligned}
& H_{w}^{f}=\frac{k_{F \uparrow}+k_{F \downarrow}}{2 m \pi \alpha} \int \mathrm{d} z \frac{\operatorname{sech}[z / L]}{L} \sin \left[\sqrt{2 \pi} \theta_{S}(z)\right] \sin \left[z\left(k_{F \uparrow}-k_{F \downarrow}\right)+\sqrt{2 \pi} \phi_{S}(z)\right] \\
& H_{w}^{b}=\frac{k_{F \uparrow}-k_{F \downarrow}}{2 m \pi \alpha} \int \mathrm{d} z \frac{\operatorname{sech}[z / L]}{L} \sin \left[\sqrt{2 \pi} \theta_{S}(z)\right] \sin \left[-z\left(k_{F \uparrow}+k_{F \downarrow}\right)+\sqrt{2 \pi} \phi_{c}(z)\right] \\
& H_{1 \perp}=\frac{2}{(2 \pi \alpha) 2} \int \mathrm{d} z \cos \left[2 \sqrt{2 \pi} \phi_{S}(z)\right]\left[g_{1 \perp \uparrow} e^{-2 i z\left(k_{F \uparrow}-k_{F \downarrow}\right)}+g_{1 \perp \downarrow} e^{2 i z\left(k_{F \uparrow}-k_{F \downarrow}\right)}\right] .
\end{aligned}
$$

We have both forward and backward scattering terms, $H_{w}^{f}$ and $H_{w}^{b}$ respectively. We also find an oscillating sine-Gordon interaction term $H_{1 \perp}$, describing "slow" Umklapp processes which are not averaged out. The normal modes of this $S U(2)$ asymmetric model have no clear physical interpretation, in contrast to the non-spin split case in which they represent pure spin and charge modes. The Hamiltonian of equation (53) can be diagonalized to find the appropriate normal modes.

Standard renormalization group analysis on the scattering Hamiltonians $H_{1}, H_{w}^{f}$ and $H_{w}^{b}$ yields the scaling equations of the form

$$
\frac{d g_{1 \perp \sigma}}{d l}=g_{1 \perp \sigma}\left[2-\gamma_{1}\right] .
$$

$\gamma_{1}$, and also the $\gamma_{f, b}$ used below, are known, but rather complicated, functions of $v_{F \sigma}, g_{2} \| \sigma$, $g_{2 \perp}, g_{4 \| \sigma}, g_{4 \perp}, g_{1 \| \sigma}$, and $g_{1 \perp \sigma}$ which can be found in the appropriate references. This first term is an irrelevant perturbation for any realistic microscopic model. Next we define the bare scattering coupling constants $g_{f / b} \equiv k_{F \uparrow} \pm k_{F \downarrow}$. For forward and backward scattering we find: 


$$
\frac{d g_{f, b}}{d l}=g_{f, b}\left[2-\gamma_{f, b}\right]
$$

In this case we find a relevant forward scattering term. However, the backward scattering term can be either relevant or irrelevant depending on the particular microscopic model used. If backward scattering is relevant then we find the usual spin-charge gapped insulating behaviour of an impurity in a Luttinger liquid. However, if only the forward scattering is relevant the spin mode is gapped, but the charge mode remains ungapped (C1S0 phase). Physically, this means that electrons no longer undergo a change of spin on passing through the domain wall. The spin of the conduction electrons becomes locked.

Due to the extended nature of the domain wall we must consider a hierarchy of length scales, as well as the relevance of the renormalized operators. Let us define $\lambda_{ \pm}=\left(k_{F \uparrow} \pm k_{F \downarrow}\right)^{-1}$, which are the relevant lengthscales for the forward and backward scattering components. From the above considerations we have several possibilities. Firstly we can have backward scattering as either relevant or irrelevant. Secondly we must take into account the relative length scales. If $\lambda_{+}<L$ then at some stage of the renormalization process (i.e. when the appropriate length scale cut-off becomes of order $\lambda_{+}$) the backward scattering terms will become small due to averaging over their oscillations. A similar case holds for the forward scattering with $\lambda_{+} \rightarrow \lambda_{-}$in the preceding. For the case in which both the forward and backward scattering lengthscales are shorter than the domain wall length we end up in the completely adiabatic limit as one would expect. However we can also be in the opposite regime when both forward and backward scattering lengthscales are longer than the domain wall length in which case one need only consider the relevancy of the appropriate operators. Note that this is possible without requiring a delta function like domain wall profile. In addition we can of course be in the intermediate regime where the backward scattering is prone to this averaging out, but the forward scattering is not. As such the low temperature fixed point depends not only on the relevance of the operators, but also on the hierarchy of length scales, and leaves three possibilities: firstly a spin-charge insulator, secondly a spin gapped charge conducting phase, and thirdly a ferromagnetic Luttinger liquid with an adiabatically behaving domain wall.

\section{Current-induced interaction of multiple domain walls}

Now consider the Hamiltonian of a one-dimensional wire in a spatially non-uniform magnetization profile $\mathbf{M}(z)$

$$
H=\int d z \psi_{\alpha}^{\dagger}(z)\left[-\frac{\partial_{z}^{2}}{2 m} \delta_{\alpha \beta}-J \boldsymbol{\sigma}_{\alpha \beta} \cdot \mathbf{M}(z)\right] \psi_{\beta}(z),
$$

and apply the local gauge transformation as described in Sec. 1. The transformed Hamiltonian can be presented as

$$
H=\int d z \psi_{\alpha}^{\dagger}(z)\left[-\frac{\partial_{z}^{2}}{2 m} \delta_{\alpha \beta}+U_{\alpha \beta}(z)-J M \sigma_{\alpha \beta}^{z}\right] \psi_{\beta}(z)
$$

where the DW-induced perturbation is

$$
U(z)=-\frac{1}{2 m}\left[2 A \partial_{z}+\left(\partial_{z} A\right)+A^{2}\right],
$$


and $A(z)=T^{\dagger}(z) \nabla_{z} T(z)$ is a gauge potential. For a wire with two DWs we can parametrize the magnetization profile by the angles $\varphi(z)$ and $\theta(z)$ (see Fig. 4)

$$
\begin{array}{r}
\mathbf{n}(z)=(\cos \theta \sin \varphi, \sin \theta \sin \varphi, \cos \varphi), \\
\varphi(z)=\underbrace{\cos ^{-1}\left(\tanh \left[\frac{z}{L}\right]\right)}_{=-\varphi_{1}(z)}+\underbrace{\cos ^{-1}\left(\tanh \left[\frac{z-z_{0}}{L}\right]\right)}_{=-\varphi_{2}(z)} .
\end{array}
$$

The angle $\theta(z)$ describes the relative orientation between the wall pinned at $z=0$ and the other situated around $z=z_{0}$. We set $\theta_{1}$ to zero at the first wall and $\theta_{2}=\theta_{0}$ around the second. For $\theta_{0}=\pi$ the walls are antialigned. For $z_{0} \leq L$ DWs may merge, hence we consider the case $z_{0}>L$ for which we may write $U(z) \approx U_{1}(z)+U_{2}(z)$, where $(j=1,2)$

$$
U_{j}(z)=\frac{\left[\varphi_{j}^{\prime}(z)\right]^{2}}{8 m}+i \sigma^{y}\left[\frac{\varphi_{j}^{\prime \prime}(z)}{4 m}+\frac{\varphi_{j}^{\prime}(z) \partial_{z}}{2 m}\right] \cos \theta_{j}-i \sigma^{x}\left[\frac{\varphi_{j}^{\prime \prime}(z)}{4 m}+\frac{\varphi_{j}^{\prime}(z) \partial_{z}}{2 m}\right] \sin \theta_{j}
$$

This approach is generalizable to any number of DWs, which are sufficiently far apart. For $k_{F} L \geq 1$, i.e. when $\mathbf{M}(z)$ hardly varies within $k_{F}^{-1}$ (adiabatic DW), the terms in Eq. (64) proportional to $\varphi_{1}^{\prime \prime}(z)$ are negligibly small and the perturbative approach is appropriate for treating the electron scattering from the DWs potential (Eq. (64)). In fact, it can be shown that this approach is justifiable even for $k_{F} L=1$. Assuming $\psi^{0}(z)$ to be the wave function of an independent electron with energy $\varepsilon$ in the wire without the DWs, we find the first-order correction due to the perturbation $U_{1}(z)$, i.e. due to scattering from the first DW, as

$$
\delta \psi_{\varepsilon}(z)=\int_{-\infty}^{\infty} d z^{\prime} G_{\varepsilon}\left(z, z^{\prime}\right) U_{1}\left(z^{\prime}\right) \psi^{0}\left(z^{\prime}\right)
$$

The Green's function $G_{\varepsilon}$ corresponds to the unperturbed Hamiltonian with $U(z)=0$. It is diagonal in spin space with elements

$$
G_{\varepsilon \sigma}\left(z, z^{\prime}\right)=-\frac{i m}{k_{\sigma}} e^{i k_{\sigma}\left|z-z^{\prime}\right|}
$$

where $k_{\sigma} \approx k_{\sigma}^{0}+\frac{i}{2 \tau_{\sigma}} \frac{m}{k_{\sigma}^{0}}$ for lifetimes $\tau_{\sigma} \gg \varepsilon_{F}^{-1}$, and $k_{\sigma}^{0}=[2 m(\varepsilon+\mu \pm J M)]^{1 / 2}$. Hence

$$
\begin{aligned}
& \delta \psi_{\varepsilon \uparrow}(z)=\int_{-\infty}^{\infty} d z^{\prime}\left(\begin{array}{c}
-\frac{i}{8 k_{\uparrow}} e^{i k_{\uparrow}\left|z-z^{\prime}\right|}\left[\varphi_{1}^{\prime}(z)\right]^{2} e^{i k_{\uparrow} z^{\prime}} \\
-\frac{k_{\uparrow}}{2 k_{\downarrow}} e^{i k_{\downarrow}\left|z-z^{\prime}\right|} \varphi_{1}^{\prime}(z) e^{i k_{\uparrow} z^{\prime}}
\end{array}\right) \\
& \delta \psi_{\varepsilon \downarrow}(z)=\int_{-\infty}^{\infty} d z^{\prime}\left(\begin{array}{c}
\left.\frac{k_{\downarrow}}{2 k_{\uparrow}} e^{i k_{\uparrow}\left|z-z^{\prime}\right|} \varphi_{1}^{\prime}(z)\right] e^{i k_{\downarrow} z^{\prime}} \\
-\frac{i}{8 k_{\downarrow}} e^{i k_{\downarrow}\left|z-z^{\prime}\right|}\left[\varphi_{1}^{\prime}(z)\right]^{2} e^{i k_{\downarrow} z^{\prime}}
\end{array}\right)
\end{aligned}
$$

for incoming electrons of spin up and down, respectively. The interaction energy of the two DWs due to the single scattered state $\psi_{\varepsilon \sigma}(z)=\psi_{\varepsilon \sigma}^{0}(z)+\delta \psi_{\varepsilon \sigma}(z)$ is calculated as

$$
\Delta E_{\sigma}=\int_{-\infty}^{\infty} d z \delta \psi_{\varepsilon \sigma}^{\dagger}(z) U_{2}(z) \delta \psi_{\varepsilon \sigma}(z) .
$$




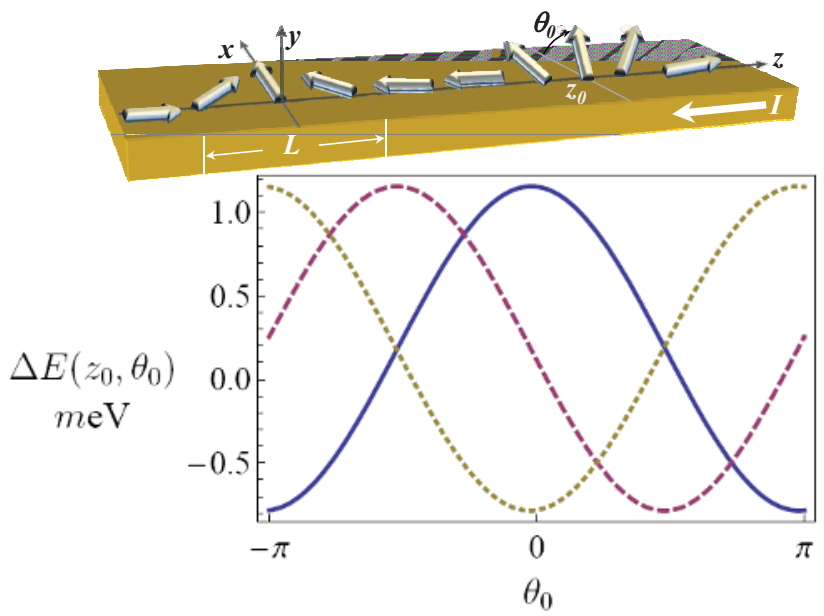

Fig. 4. Top panel: A schematics showing the DWs magnetization profile (thick arrows). $L$ is the DW width, $z_{0}$ and $\theta_{0}$ are respectively the DW position and orientation with respect to the DW at $z=0, I$ is the current. Lower panel: Interaction energy $\Delta E\left(z_{0}, \theta_{0}\right)$ as a function of $z_{0}$ and $\theta_{0}$. Solid curve is for $z_{0}=3.67 \mathrm{~nm}$, the dashed is for $z_{0}=4.13 \mathrm{~nm}$, and the dotted is for $z_{0}=4.40 \mathrm{~nm}$.

Summing up the contributions of all scattering states in the energy range between $\varepsilon_{F}$ and $\varepsilon_{F}+e \Delta \phi / 2$, for an applied voltage $e \Delta \phi / 2 \ll \varepsilon_{F}$, we obtain the current-induced coupling of the DWs as

$$
\Delta E=\frac{e \Delta \phi}{\sqrt{2} \pi}\left(\frac{\Delta E_{\uparrow}}{v_{\uparrow}}+\frac{\Delta E_{\downarrow}}{v_{\downarrow}}\right)
$$

where $v_{\sigma}=k_{\sigma}^{0} / m$ is the velocity of electrons at the Fermi level.

Figure 4 shows the dependence of the coupling energy $\Delta E$ as a function of distance $z_{0}$ and relative angle $\theta_{0}$ of the second DW. In the calculations we used the parameters: $\lambda_{F}=0.367 \mathrm{~nm}$ (Fe), $\tau_{\sigma}^{-1}=10^{-9} \varepsilon_{F}, L=\lambda_{F}, J M=0.2 \varepsilon_{F}, \varepsilon_{F}=11.2 \mathrm{eV}$, and $e \Delta \phi=0.01 \varepsilon_{F}$ corresponding to an applied voltage of $112 \mathrm{meV}$. As we see, the interaction energy depends periodically on the DWs mutual angle $\theta_{0}$ and distance $z_{0}$, which results in an oscillating motion of the DW along the axis $z$ as well as an oscillating direction of DW polarization.

Now we focus on the effect of DW scattering on the electron spin density, leading to a nonequilibrium spin accumulation and to a spin torque acting on the wall. Subsequently, we study the dynamics of the DW related to the DW coupling. The spin-density due to the single transmitted wave of spin $\sigma$ is

$$
\mathbf{S}_{\sigma}(z)=\psi_{\varepsilon \sigma}^{\dagger}(z) T(z) \boldsymbol{\sigma} T^{\dagger}(z) \psi_{\varepsilon \sigma}(z),
$$

and the total current-induced spin density is

$$
\mathbf{S}(z)=\frac{e \phi}{2 \pi}\left(\frac{\mathbf{S}_{\uparrow}}{v_{\uparrow}}+\frac{\mathbf{S}_{\downarrow}}{v_{\downarrow}}\right) .
$$




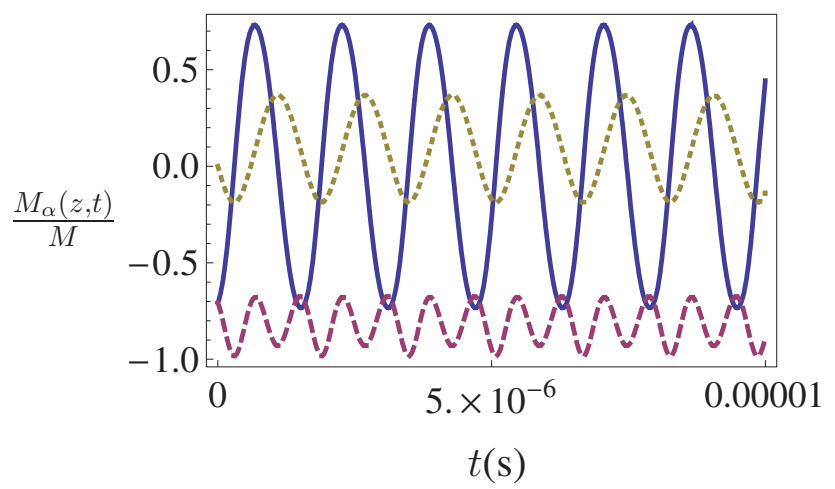

Fig. 5. The time dependence of the magnetization with the initial condition for the second wall to be at an angle of $\theta_{0}=\pi / 4$ to the first wall. This is the solution to Eq. 74 with $M \approx 5.56 \times 10^{4} \mathrm{Am}^{-1}$. The solid curve is the $x$-component, dashed the $y$-component, and dotted the $z$-component. Taken at the centre of the domain wall.

We find that the correction to the spin density follows the magnetization profile with additional Friedel oscillations, which are a superposition of two waves with periods $k_{F \uparrow}^{-1}$ and $k_{F \downarrow}^{-1}$. The oscillations in the spin density are smaller in magnitude than the overall spin density profile. One can also calculate the current-induced torque acting on the second DW at $z$ from

$$
\Delta \mathbf{T}\left(z, z_{0}, \theta_{0}\right)=-\frac{\gamma J}{\sigma_{\mathcal{C S}}} \mathbf{M}\left(z, z_{0}, \theta_{0}\right) \times \Delta \mathbf{S}\left(z, z_{0}, \theta_{0}\right),
$$

where $\gamma=g \mu_{B}, g$ is the Landé factor and $\mu_{B}$ is the Bohr magneton. The results of numerical calculations show that the force upon one of the DWs from the other one depends strongly on their relative polarizations.

Thus, the current through a magnetic nanowire containing DWs results in a DW interaction mediated by the scattered charge carriers. We developed a method for calculating the interaction energy and the consequences of this new coupling mechanism. The DWs interaction energy oscillates as a function of the DWs mutual orientation and distance. This has immediate consequences on how DWs rearrange upon applying a bias voltage and on the fundamental limit of the DWs packing density. In fact, different parts of the DW oscillate at different rates and in different ways: becoming more regular, smaller, and quicker away from the DW centre. This effect will be modified by the exchange interaction for longer domain walls, where there is an obvious limit to how much one can distort the domain wall before the energy costs becomes too high. The nonequilibrium DWs oscillations around the energy minima generates radiation with a frequency dependent on the applied bias voltage, DW length and scattering strength. These parameters are externally tunable for utilizing the interacting DWs as a versatile radiation source.

The effect of the modified spin density, which is the cause of the current induced interaction between the DWs, on the motion of the DW can be found using the standard Landau-Lifschitz equation (Lifschitz (1980)). To inspect the current-induced dynamics of the DW at $z=z_{0}$, we evaluate the accumulated spin density that acts on the DW at $z=z_{0}$. Thus for a relatively sharp domain wall which can be effectively treated as a magnetic moment we have only to 
solve, at $z=z_{0}$ :

$$
\partial_{t} \mathbf{M}=-\frac{\gamma J}{\sigma_{\mathcal{C S}}} \mathbf{M} \times \mathbf{S}[\mathbf{M}] .
$$

As an initial condition we assume that the magnetization profile in the wire without electric current is described by Eq. (63). The results for the time dependence of the magnetization are shown in Fig. 5 for the centre of the DW, $z=z_{0}$. We should note that the relative orientation of the walls at the start of motion does play a role in the type of motion we see. Here we present it for an arbitrary configuration, $\theta_{0}=\pi / 4$. The DW motion is a result of the excess spin torque generated by the first DW, which is felt as an effective applied magnetic field. As such precession, as demonstrated in Fig. 5, is precisely what one would expect. The crucial point is that the characteristics of this precession depend upon the distance between, and relative orientation of, the two DWs.

The extension of our analysis to include the effects of magnetic anisotropy is straightforward, and constrains the motion in the appropriate direction. If one also wishes to consider the motion of a longer domain wall it is no longer possible to neglect the exchange interaction. In such a scenario one still finds distortion of the domain wall, but due to the extended nature of the spin density correction, it cannot produce any coherent motion. However, when one considers current induced motion (Zhang et al. (1980)) these spin density terms can allow for motion with smaller applied currents. This spin density takes on the role of an applied magnetic field, which is well known to ease the current induced motion of domain walls.

\section{Conclusions}

Using the presented models of electrons moving in a spatially varying magnetization field, one can study the properties of magnetic domain walls in magnetic nanowires. This allows for the calculations of charge and spin currents, current-induced spin torque, and current-induced interactions in magnetic nanowires. These are characteristics that strongly depend on the presence of domain walls. Our considerations were predominantly concentrated on very thin domain walls, where presence of a domain wall may affect very strongly the transmission of electrons in nanowires. Experimentally such thin domain walls can appear for instance in nanoconstrictions (Bruno (1999)). Domain walls in magnetic semiconductors with relatively low carrier density can be considered as thin because the electron wavelength in semiconductors $\lambda$ is usually of the same order of magnitude or larger than the width of the walls.

\section{Acknowledgement}

This work is supported by the DFG through contract BE 2161/5-1 and SFB762, FCT Grant No. PTDC/FIS/70843/2006 in Portugal, Polish Ministry of Science and Higher Education as a research project in years 2011 - 2013 and by the Graduate School of MAINZ (MATCOR).

\section{References}

Araújo, M.A.N.; Dugaev, V.K.; Vieira, V.R.; Berakdar, J. \& Barnaś, J. (2006) Transmission of correlated electrons through sharp domain walls in magnetic nanowires: a renormalization group approach. Physical Review B, Vol. 74, No. 22, (December 2006) 224429 
Araújo, M.A.N.; Berakdar, J.; Dugaev, V.K. \& Vieira, V.R. (2007). Role of a spin-flip scatterer in a magnetized Luttinger liquid. Physical Review B, Vol. 76, No. 20, (November 2007) 205107

Bruno, P. (1999). Geometrically constrained magnetic wall. Physical Review Letters, Vol. 83, No. 12, (September 1999) 2425-2428

Cabrera, G.G. \& Falicov, L.M. (1974). Theory of the residual resistivity of Bloch walls I. Paramagnetic effects. Physica Status Solidi B, Vol. 61, No. 2, (February 1974) 539-549

Cabrera, G.G. \& Falicov L.M. (1974). Theory of the residual resistivity of Bloch walls. II. Inclusion of diamagnetic effects. Physica Status Solidi B, Vol. 62, No. 1, (March 1974) 217-222

Chopra, H.D. \& Hua, S.Z. (2002). Ballistic magnetoresistance over 3000\% in Ni nanocontacts at room temperature. Physical Review B, Vol. 66, No. 2, (June 2002) 020403(R)

Dugaev, V.K.; Barnaś, J.; Łusakowski, A. \& Turski, L. (2002). Electrons in a ferromagnetic metal with a domain wall. Phys. Rev. B, Vol. 65, No. 22, (June 2002) 224419

Dugaev, V.K.; Berakdar, J. \& Barnaś, J. (2003). Reflection of electrons from a domain wall in magnetic nanojunctions. Physical Review B, Vol. 68, No. 10, (September 2003) 104434

Dugaev, V.K.; Berakdar, J. \& Barnaś, J. (2004). Tunable conductance of magnetic nanowires with structured domain walls. Physical Review Letters, Vol. 96, No. 4, (February 2004) 047208

Dugaev, V.K.; Barnaś, J.; Berakdar, J., Ivanov, V.I.; Dobrowolski, W. \& Mitin, V.F. (2005). Magnetoresistance of a semiconducting magnetic wire with a domain wall. Physical Review B, Vol. 71, No. 2, (January 2005) 024430

Dugaev, V.K.; Vieira, V.R.; Sacramento, P.D.; Barnaś, J.; Araújo, M.A.N. \& Berakdar, J. (2006). Current-induced motion of a domain wall in a magnetic nanowire. Physical Review B, Vol. 74, No. 5, (August 2006) 054403

Dugaev, V.K.; Vieira, V.R.; Sacramento, P.D.; Barnaś, J.; Araújo, M.A.N. \& Berakdar, J. (2007). Current-induced spin torque on a domain wall in a magnetic nanowire. International Journal of Modern Physics, Vol. 21, Nos. 8 \& 9, (April 2007) 1659-1663

Ebels, U.; Radulescu, A.; Henry, Y.; Piraux, L. \& Ounadjela, K. (2000). Spin accumulation and domain wall magnetoresistance in $35 \mathrm{~nm}$ Co wires. Physical Review Letters, Vol. 84, No. 5, (January 2000) 983-986

Kläui, M. (2008). Head-to-head domain walls in magnetic nanostructures. Journal of Physics: Condensed Matter, Vol. 20, No. 31, (August 2008), 313001

Lifshitz, E.M. \& Pitaevskii L.P. (1980). Statistical Physics Part 2: Theory of the Solid State Butterworth-Heinemann, ISBN-10 0750626364, Oxford

Matveev, K.A.; Yue, D. \& Glazman , L.I. (2000). Tunneling in one-dimensional non-Luttinger electron liquid. Physical Review Letters, Vol. 71, No. 20, (November 1993) 3351-3354

Parkin, S.S.P.; Hayashi, M. \& Thomas, L. (2008). Magnetic domain-wall racetrack memory. Science, Vol. 320, (April 2008) 190-194.

Penc, K. \& Sólyom, J. (1993) One-dimensional Hubbard model in a magnetic field and the multicomponent Tomonaga-Luttinger model. Physical Review B, Vol. 47, No. 11, (1993) 6273-6292

Rüster, C.; Borzenko, T.; Gould, C.; Schmidt, G.; Molenkamp, L.W.; Liu, X.; Wojtowicz, T.J.; Furdyna, J.K.; Yu, Z.G. \& Flatté, M.E. Very large magnetoresistance in lateral ferromagnetic (Ga,Mn)As wires with nanoconstrictions. Physical Review Letters, Vol. 91, No. 21, (November 2003) 216602 
Sedlmayr, N.; Dugaev, V.K.; \& Berakdar, J. (2009). Current-induced interactions of multiple domain walls in magnetic quantum wires. Physical Review B, Vol. 79, No. 17, (May 2009) 174422

Sedlmayr, N.; Dugaev, V.K.; \& Berakdar, J. (2010). Role of non-collinear magnetization: From ferromagnetic nanowires to quantum rings. Physica Status Solidi B, Vol. 247, No. 10, (August 2010) 2603-2609

Sedlmayr, N.; Dugaev, V.K.; Berakdar, J.; Vieira, V.R.; Araújo, M.A.N.; \& Barnaś, J. (2010). Spin and charge transport through non-collinear magnetic nanowires. Journal of Magnetism and Magnetic Materials, Vol. 322, Nos. 9-12 (May-June 2010) 1419-1421

Sedlmayr, N.; Eggert, S. \& Sirker, J. (2011). Electron scattering from domain walls in ferromagnetic Luttinger liquids. cond-mat.str-el arXiv:1102.5446 (February 2011).

Tatara, G. \& Fukuyama, H. (1997). Resistivity due to a domain wall in ferromagnetic metal. Physical Review Letters, Vol. 78, No. 19, (May 1997) 3773-3776

Zhang, S. \& Li, Z. (2004). Roles of nonequilibrium conduction electrons on the magnetization dynamics of ferromagnets. Physical Review Letters, Vol. 93, No. 12, (September 2004) 127204 


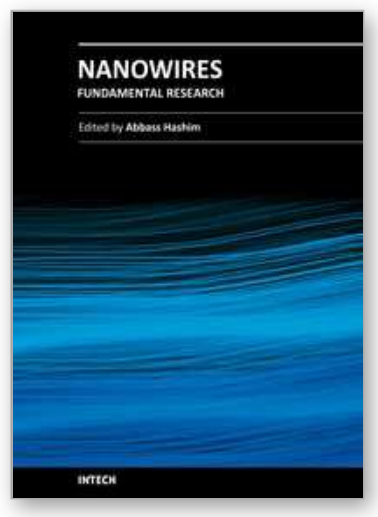

\author{
Nanowires - Fundamental Research \\ Edited by Dr. Abbass Hashim
}

ISBN 978-953-307-327-9

Hard cover, 552 pages

Publisher InTech

Published online 19, July, 2011

Published in print edition July, 2011

Understanding and building up the foundation of nanowire concept is a high requirement and a bridge to new technologies. Any attempt in such direction is considered as one step forward in the challenge of advanced nanotechnology. In the last few years, InTech scientific publisher has been taking the initiative of helping worldwide scientists to share and improve the methods and the nanowire technology. This book is one of InTechâ $€^{\mathrm{TM}_{\mathrm{S}}}$ attempts to contribute to the promotion of this technology.

\title{
How to reference
}

In order to correctly reference this scholarly work, feel free to copy and paste the following:

Jamal Berakdar, Vitalii Dugaev, Jozef Barnas and Nicholas Sedlmayr (2011). Charge and Spin Transport in Magnetic Nanowires, Nanowires - Fundamental Research, Dr. Abbass Hashim (Ed.), ISBN: 978-953-307-3279, InTech, Available from: http://www.intechopen.com/books/nanowires-fundamental-research/charge-andspin-transport-in-magnetic-nanowires

\section{INTECH}

open science | open minds

\section{InTech Europe}

University Campus STeP Ri

Slavka Krautzeka 83/A

51000 Rijeka, Croatia

Phone: +385 (51) 770447

Fax: +385 (51) 686166

www.intechopen.com

\section{InTech China}

Unit 405, Office Block, Hotel Equatorial Shanghai

No.65, Yan An Road (West), Shanghai, 200040, China 中国上海市延安西路65号上海国际贵都大饭店办公楼 405 单元 Phone: $+86-21-62489820$

Fax: +86-21-62489821 
(C) 2011 The Author(s). Licensee IntechOpen. This chapter is distributed under the terms of the Creative Commons Attribution-NonCommercialShareAlike-3.0 License, which permits use, distribution and reproduction for non-commercial purposes, provided the original is properly cited and derivative works building on this content are distributed under the same license. 